\title{
Sistema de innovación tecnológica desde la perspectiva del desarrollo endógeno. Reflexión teórica
}

\author{
Benavides de Finol, Luisa* \\ Guzmán Parra, Vanesa* \\ Vila Oblitas, José ${ }^{* * *}$
}

\begin{abstract}
Resumen
El propósito de esta investigación es identificar los componentes estructurales de un sistema de innovación tecnológica en el marco del desarrollo endógeno (propuesto por el Gobierno de Venezuela) que permita, reinterpretar desde una nueva perspectiva el rol de la innovación. La estrategia metódica se centró en la revisión y análisis de la literatura actualizada sobre el tema, de la información proporcionada por expertos calificados y por actores participantes en procesos de innovación tecnológica. El tratamiento de la información recabada se hizo desde la perspectiva teórico-metodológica de sistemas sociales de Luhmann (2007). Las conclusiones se expresan en la definición de los subsistemas propuestos y la explicación del papel que debe jugar cada uno de ellos en el sistema de innovación basado en los principios de desarrollo endógeno. Los cuatro subsistemas son: tejido social, marco institucional, proceso de innovación y el actor social o emprendedor en la empresa, los cuales se dinamizan en los entornos interno y externos y deben ser dinamizados en cada territorio con sus particularidades.
\end{abstract}

Palabras clave: Innovación tecnológica; aprendizaje; desarrollo endógeno sustentable; sistema social.

Recibido: 10.02.14. Aceptado: 19.05.15

* Doctora en Economía y Administración de Empresas Universidad de Málaga. España, Socióloga y Profesora de la Universidad Ezequiel Zamora. Venezuela. Correo: luisa.benavides@gmail. com

** Doctor y Profesor del Departamento de Economía y Administración de Empresas de la Universidad de Málaga. España. Correo: fparra@uma.es

*** Doctora y Profesora del Departamento de Economía y Administración de Empresas de la Universidad de Málaga. España. Correo:vgp@uma.es 


\title{
Technological innovation system from the endogenous development perspective. Theoretical reflection
}

\begin{abstract}
The aim of this study is to identify the structural components of a technological innovation system within the framework of endogenous development (proposed by the Venezuelan Government) that allows to reinterpret from a new perspective the role of the innovation. The methodic strategy was centered in revision and analysis of the actualized on topic literature, the information proportioned by qualified experts and participating actors in the technological innovation process. The information processing was made from Luhmanns (2007) theoretical-methodological perspective of social systems. The conclusions are expressed on the proposed subsystems definition and the explanation of the role each one of them must play in the innovation system based on endogenous development principles. The four subsystems are: Social fabric, institutional framework, innovation process and the social actor or entrepreneur in the company, boosting them in the internal and external environment and must be stimulated in every territory with their special features.
\end{abstract}

Key Words: Technological innovation; apprenticeship; sustainable endogenous development; social system.

\section{Introducción}

La expansión del capitalismo y sus consecuencias para los países periféricos, como la globalización, han alterado sustancialmente sus procesos de desarrollo, a la vez que han descolocado los elementos de su dinámica económica y generando efectos negativos para la sociedad en su conjunto.

Una recolocación de dichos elementos con la finalidad de mejorar estos resultados obliga a considerar que, tal como aseguran Cabello, Ortiz (2013) debe concretarse una intervención estratégica desde el Estado para el fomento de la innovación y el desarrollo científico y tecnológico, a fin de promover una economía más competitiva. Para ello es importante mejorar el aparato productivo del estado por lo que se sugiere impulsar políticas púbicas en la que esté considerado el tema de la innovación.
El decreto $\mathrm{N}^{\circ} 3895$ sobre el desarrollo endógeno y empresas de producción social, emitido desde la Presidencia de la República de Venezuela (2005), tiene la finalidad de fortalecer el sector productivo nacional, a través de un desarrollo alternativo desde lo local, en este modelo la innovación es un factor central sobre todo si se apoya en los principios que definen el desarrollo endógeno. El desarrollo endógeno y la innovación fundamentada en los principios que lo definen llevan a rescatar no solo las estrategias hacia lo económico sino hacia la mejora de la cultura en general. La urgencia de entender el desarrollo como un proceso en el cual habría que tener en cuenta permanentemente la satisfacción de las necesidades humanas, va más allá de la riqueza material.

Esta idea se ve reflejada claramente en la Organización de Estados Iberoamericanos para la Educación, la 
Ciencia, la Tecnología y la Cultura (2012) cuando hace mención a los índices de Desarrollo Humano definitorios de la calidad de vida, los cuales se concretan en el acceso a la educación, la vivienda y los servicios públicos. Visto así los países deberían garantizar un mínimo de satisfacción a su población para sacarlas del subdesarrollo, puesto que el desarrollo no es sinónimo de riqueza, sino de competencia gerencial en la administración pública de sus bienes activos.

El interés prioritario del desarrollo entonces, debería ser incrementar las capacidades humanas a partir de las cuales deja de convertirse en una meta para transformarse en un proceso permanente de aprendizaje social. Puesto en términos más simples, es hacer del ser humano (sujeto y objeto) el centro del desarrollo (Slee, 2003).

Los años que sucedieron a las guerras mundiales crearon grandes esperanzas para estos países que se vieron reflejadas en políticas de desarrollo para América Latina, sin embargo como lo afirma Albornoz (2002), los resultados no han sido los esperados. La idea central del trabajo considera que los errores cometidos en la aplicación de las políticas de desarrollo y más concretamente de las políticas de desarrollo alternativo, en este caso de desarrollo endógeno relacionado con la innovación, deben ser capitalizados para plantear opciones pertinentes y sustentables capaces de ser manejadas desde adentro. Así se podrán promover las acciones de autoevaluación, de autodefinición y readaptabilidad. Este sistema deberá ser accesible y de fácil manejo. Los actores involucrados en los procesos de producción podrán materializar la optimización de las actividades económicas productivas mediante la innovación (Arocena, 2002).

Se entiende como requisito para el proceso de desarrollo alternativo la innovación como factor clave en un entorno favorable a la creación de iniciativas, una red institucional que propicie tal proceso, un marco legal que lo viabilice y actores sociales en proceso permanente de aprendizaje que lo haga sustentable.

La metodología utilizada se enmarca en un diseño documental completado con la técnica de recolección de datos a través de entrevistas no estructuradas a expertos en el tema. Esto permitió entender la correspondencia esperada entre innovación y el marco de referencia concretado en el modelo de desarrollo endógeno, propuesto por el gobierno de Venezuela desde el Ministerio de la Economía Popular, a través de los aportes de Lanz (2004) y Jaua (2005). Igualmente se ha revisado la bibliografía pertinente así como la opinión de los expertos que concluyen que las empresas en lberoamérica, realizan poca inversión en innovación y desarrollo (Albornoz, 2002).

El desarrollo endógeno alternativo es por naturaleza local y sustentable, esto requiere un tipo específico de innovación con unas características que deben estar orientadas a fortalecer la independencia de las regiones. Esta idea representa el leit motiv de esta investigación (Lanz, 2004).

El presente trabajo tiene como finalidad identificar los componentes de un sistema de innovación tecnológica en el marco del desarrollo endógeno que permita reinterpretar desde una nueva perspectiva el rol de la innovación en el desarrollo local. 
Sistema de innovación tecnológica desde la perspectiva del desarrollo...

Benavides de Finol, Luisa, Guzmán Parra, Vanesa y Vila Oblitas, José

\section{Técnica, tecnología e innovación}

Los conceptos de técnica, tecnología e innovación tienen una gran pertinencia en la reflexión sobre la innovación tecnológica. El hombre utilizó la tecnología para modificar y transformar la materia bruta que le ofrecía la naturaleza en herramientas o piezas utilitarias haciendo entonces que tenga una representación física, tangible y útil; aunque para estos tiempos la tecnología pasó también al plano de lo intangible.

En este sentido, la tecnología podría entenderse como el conjunto ordenado de conocimientos, procesos y procedimientos cuyo objeto es el de producir bienes de uso y de consumo que satisfagan la calidad de vida del hombre y en virtud de ello corresponden a las necesidades de su época, de allí que exista una relación directa entre el conocimiento, la investigación y el desarrollo (Vega, 2012).

Por otro lado la técnica se asocia a la tecnología y es concebida como el resultado de la aplicación de un buen conocimiento tecnológico. Mientras que la tecnología crea y mejora lo existente, la técnica repite sin aportar mejoras a lo desarrollado. La tecnología requiere de un saber profundo y maduro sobre la naturaleza de los recursos y explica la importancia de la ciencia, como fuente de saber tecnológico y hace uso de conocimientos y competencias.

El tema de la innovación es muy complejo, contiene múltiples conceptualizaciones que responden a diversas disciplinas, paradigmas e ideología donde encuentra las bases para ser definido (Quintero y López, 2012). Como consecuencia de lo expresado anteriormente se desprende que existen diversas formas de plantearlo. En este caso, se hizo desde la perspectiva del desarrollo endógeno que adicionalmente tiene una serie de principios de orden socio-político que lo orientan pero también dificultan su aplicación (Vázquez-Barquero, 1997).

Así mismo la innovación tecnológica es un concepto que puede ser definido desde distintas perspectivas, en este caso interesa definirlo, tal como lo apuntó Schumpeter (1934), como la introducción de un nuevo producto, un nuevo método de producción, la apertura de un nuevo mercado, la de una nueva fuente de materia prima, la puesta en práctica de una organización en alguna industria, puede ser el acomodo a cualquier crisis. De igual forma lo apunta López (1997), quien sostiene que la innovación en las empresas es la fuente de transformación de una idea o un nuevo producto de allí que las nuevas ideas son el desencadenante del proceso innovador convirtiéndose en su materia prima. Se enfocará entonces a partir de la creencia de que el elemento humano de la sociedad tiende a innovar por naturaleza lo que lo coloca en ventaja frente a su propia realidad.

Esta reflexión está enfocada hacia la innovación local, la cual ha sido vista como una forma de solucionar problemas de todo tipo en los espacios locales, con la idea de fortalecer las regiones e incorporar la innovación en los procesos productivos desde la agroindustria rural familiar, hasta los industriales en las pequeñas y grandes empresas. Sin embargo, a pesar de que el concepto de innovación estuvo asociado a desarrollo tecnológico y en el discurso moderno aparece como la panacea capaz de resolver todos los problemas de la sociedad, en el siglo XXI se puede 
afirmar que tal y como fue interpretado por los países ricos y productores de tecnología, puede ser más bien definido como un elemento que ha profundizado las inequidades sociales y la brecha entre unos y otros.

Esta concepción de innovación, plantea la necesidad de revertir tales efectos, por lo cual es preciso mirar su desempeño desde diferentes perspectivas si se quiere involucrar en los reales procesos de desarrollo a los países emergentes, entre ellos los de América Latina. Los países en desarrollo son como bien los define Arocena (2002), aquellos caracterizados por su heterogeneidad, situación que imposibilita su clasificación rigurosa, sin embargo, también hace notar su falta de ingreso desde su transición agraria a los procesos de industrialización.

Aunque después de la segunda guerra mundial se vio un florecimiento en la mayoría de los países de América Latina por las estrategias para el avance de la industrialización, las consecuencias de las prácticas implementadas en casi todos sus gobiernos, desafortunadamente no se profundizaron las acciones de innovación en el sentido de incorporarlas a la concepción del desarrollo asumida tal como lo afirma la Organización de Estados Iberoamericanos para la Educación, citada por Albornoz (2012:25).

Adicionalmente, la ausencia de sistemas de innovación en casi todos los países de América Latina interpela a los promotores del desarrollo, en el sentido de que la innovación ha sido incorporada a todos los procesos, desde una perspectiva aislada, sin considerarlo como parte fundamental en la configuración de una estructura emancipada en todos sus aspectos tal como se aspira.

Si bien es cierto que en general se manejan logros importantes en ciencia y tecnología, estos, a pesar de haber contado históricamente con apoyos financieros importantes por parte del Gobierno Nacional no han tenido los impactos esperados específicamente en el desarrollo local (Avalos, entrevista 2012).

En síntesis, se afirma que han existido intentos de incorporar el tema de la innovación, pero no han sido suficientes ni mucho menos eficientes, por lo menos en lo que atañe a países como Venezuela. El desarrollo y la innovación han sido interpretados de forma aislada (Avalos, entrevista 2012).

La reflexión en torno a la innovación como impulsora del desarrollo, remite la investigación hacia la necesidad de verla inmersa en una estructura más compleja, que permita su inserción en los procesos de desarrollo de una manera coherente.

Por lo tanto, la participación de los actores involucrados en la creación de sus propios espacios para la vida, se presenta como el elemento clave, habida cuenta de que forma parte, de los principios del desarrollo endógeno relacionados directamente al actor social.

\section{La perspectiva local como respuesta}

El desarrollo local no es un tema nuevo, ha sido abordado, entre otros, por Buarque (1999), Cuervo (1998) y Borja y Castells (1997) citados por Bossier, (2009); Touraine (2001); Calvelo (2001); Ochoa (2005); Arocena (2002), desde muchas perspectivas. En el caso de esta investigación, se ha relacionado con la participación como elemento esencial y se ha incluido en la definición de desarrollo endógeno.

El concepto de desarrollo local compartido por los autores antes mencionados, conllevan a definirlo 
Sistema de innovación tecnológica desde la perspectiva del desarrollo...

Benavides de Finol, Luisa, Guzmán Parra, Vanesa y Vila Oblitas, José

como una respuesta a los problemas de desorganización económica producida por el deterioro de los instrumentos de intervención del estado y su pérdida de eficiencia en la regulación de la economía (Vázquez-Barquero, 1997).

De allí que se piense, en el crecimiento económico, desde la perspectiva del cambio estructural de la población local, permitiendo una mayor intervención en los procesos de desarrollo global. Este proceso se da en tres dimensiones: económica, sociocultural y política administrativa. Sin embargo estas dimensiones dinamizadas en lo local deben vincularse a la economía nacional.

En este sentido Borja y Castells citados por Boissier (2009) señalan que lo global y lo local son complementarios reforzando la propuesta de un sistema vinculante entre lo local y lo central. A partir de esta premisa se diseñaron los subsistemas tomando en consideración las dimensiones de Vázquez-Barquero (1997) se distribuyeron entre los factores internos y factores externos como un aporte metodológico al diseño del sistema de innovación.

Martínez Lillo (2012) hace una clara referencia a la necesidad del apoyo institucional para promover la participación; pero sobre todo para definir los mecanismos que permitan establecer estos vínculos, desde lo local hacia lo externo. Esta afirmación, conlleva a pensar que debe haber una clara definición de lo interno y lo externo y sus formas de funcionar adecuadamente. Afirma también que no es tarea fácil pero si imprescindible.

Finalmente el aporte de Martínez Lillo (2012), visualiza el potencial de las universidades públicas locales como socios en el desarrollo y fortalecimiento de acciones de cooperación al desarrollo.
El informe Stiglitz (2013) propone dejar de lado las medidas neoliberales porque ellas han agudizado la crisis. Habría entonces que centrarse en el desarrollo local alternativo y sustentable para minimizar las desigualdades, para ello la participación de los actores sociales involucrados en los procesos también se torna importante.

Corvalán y Ferreira (2003) son mucho más tajantes cuando afirman que la participación es una metodología en el desarrollo local.

En estos entornos (locales) también se valora ampliamente el papel de la innovación, ella sería el complemento que le da fundamento a las acciones de solución de problemas in situ, no solo en los procesos productivos, sino también en las situaciones que se presentan en todos los ámbitos.

Esta búsqueda de la autonomía y la independencia del centro vincula al actor social a toda la estructura económica, desde una nueva perspectiva, fundamentalmente como impulsadores del desarrollo, lo que supone que la innovación y el desarrollo local, también llamado desarrollo endógeno, necesita un esquema metodológico y organizativo, para poder intervenir los procesos en el cual el hombre como actor social es lo fundamental.

Estos elementos, afirma Luhmann (2007), en el desarrollo de la teoría de sistemas sociales, son autopoiéticos, es decir que tienen la capacidad de autorregularse y autoevaluarse a través de los mismos actores sociales y la dinámica que propicie su funcionamiento. Esta forma de ver el problema conlleva a esperar que tal como se dinamicen los componentes, de la estructura potenciada por los actores, en relación a los contextos, más sustentable será el sistema de innovación. Esto obliga 
a darles un peso específico a los actores sociales, amén de propiciar su actualización y capacitación permanente (Leal, 2013).

En lo que se refiere a los planteamientos teóricos sobre desarrollo local que sirven de base para este estudio se menciona a Buarque,1999 (citado por Boissier, 2009), quien plantea que este tipo de estrategia económica alternativa debe experimentarse en pequeñas poblaciones $\mathrm{o}$ en pequeñas unidades territoriales para generar dinamismo económico y sobre todo ir mejorando la calidad de vida. La reflexión permitió elaborar una definición abarcadora y coherente con los planteamientos de innovación bajo las premisas de desarrollo endógeno.

El desarrollo endógeno va a ser visto como la acepción de desarrollo territorial referida a la porción más pequeña de la geografía, y es bastante similar al concepto de desarrollo local, e igualmente impreciso.

Nace como una reacción a las prácticas y líneas de pensamiento dominantes durante las décadas de los 50 y 60 , enmarcadas en el paradigma industrial fordista, y ha sido reinventado de la mano del concepto de crecimiento endógeno, propio de los nuevos modelos de crecimiento económico global, en el que la innovación tecnológica es un fenómeno interno originado en la propia función de producción, o generado desde adentro (Vázquez -Barquero, 1997).

El desarrollo debe ser considerado más y más endógeno en la medida que esté más relacionado con la cultura y los valores locales, los cuales son los que identifican en última instancia a la comunidad.

El concepto de Innovación asumido es entonces, aquel que lo entiende como técnica generada a partir de los saberes locales; para dar solución a los problemas locales de producción industrial (Boissier, 2005).

Debe ser visto de una forma holística o más bien sistémica en la que lo más importante es la manera como se dinamizan los componentes del sistema de innovación en el modelo de desarrollo alternativo.

\section{Innovación tecnológica y principios del desarrollo endógeno}

Para indagar acerca de los componentes de un sistema de innovación tecnológica bajo los principios o premisas del desarrollo endógeno, se prefiguró una estructura que da cuenta de varios subsistemas que se desprenden de los modelos teóricos que lo definen, se pueden colocar ordenadamente en tres niveles perfectamente diferenciados y complementarios, a saber: (VázquezBarquero, 1997)

a) Nivel Político: Se caracteriza porque propone un ambiente descentralizado, democrático, con la participación de todos los actores sociales de la zona y orientado hacia la mejora de la calidad de vida no solo desde el punto de vista material sino también el no material.

b) Nivel Económico: Propicia procesos productivos en pequeña y mediana escala, genera redes de producción y de consumo. Es de suma relevancia contar con bases legales de referencia que garanticen la seguridad jurídica para la inversión, la reinversión de los excedentes en el mismo sistema productivo y la generación de ganancias.

c) Nivel Tecnológico Cultural: Utiliza los saberes locales para la resolución 
Sistema de innovación tecnológica desde la perspectiva del desarrollo... Benavides de Finol, Luisa, Guzmán Parra, Vanesa y Vila Oblitas, José

de problemas, presentados en los procesos productivos, contando con recursos de la zona y donde la técnica tiene también su espacio, fortaleciendo la autonomía en relación al centro. El reconocimiento de los líderes locales cumple un papel clave para la conformación de la identidad nacional de los actores sociales y el de un buen esquema de autoestima personal.

Estos tres niveles se conformarán en dimensiones resultantes de los cuatro subsistemas que se ubican en los ámbitos externo relativo al tejido institucional y al marco de referencia legal, regulador de toda la dinámica sociocultural y económica de un país $\mathrm{y}$, el ámbito interno, relacionado a las actividades propias de la innovación tanto en la empresa, como fuera de ella, amén del compromiso de los actores sociales.

El desarrollo endógeno es una estrategia de desarrollo que debe ser vista como una organización económica alternativa que exige a cada ciudadano asumir la transformación social y esto pasa por un cambio de conciencia individual que lleve a dejar de pensar que el estado es el único garante de su bienestar (Ministerio de Planificación y Desarrollo Social, 2005).

Todos estos elementos que prefiguran la noción de innovación bajo los principios de desarrollo endógeno, no tienen ningún valor si no se dinamizan en un marco más amplio que se vincule a la economía nacional, porque de otra manera lo que propicia es el aislamiento y el regreso a las economías duales, triste experiencia del pasado en los principios de la modernización. El estado también debe garantizar un marco legal de referencia, regulador, no controlador de la actividad económica, que permita la autonomía de las regiones en lo que atañe a las competencias administrativas.

El planteamiento supone visualizar la construcción de los subsistemas de la innovación local bajo los principios de Desarrollo Endógeno, como resultado de la definición de dos tipos de factores: los externos y los internos. Entre los externos tal como hemos mencionado se encuentra el tejido social y el marco legal de referencia, mientras que en el plano interno, los procesos de innovación y los emprendedores o actores sociales involucrados

Entre los autores estudiosos del tema se encuentran: Vázquez- Barquero (1997); Touraine (2001); Lanz (2004); Jaua (2005); Calvelo (2001) y Ochoa (2005), coinciden en la conceptualización $y$ en las dimensiones o planos que la definen: el económico, el político y el tecnológico-cultural.

Hay aportes que también han enriquecido la discusión para la configuración de la estructura o sistema de innovación bajo los principios del desarrollo endógeno como los representados por la autogestión y la sustentabilidad, que se conectan esencialmente con la mejora de la calidad de la vida material, intelectual, cultural y afectiva de todas las personas $y$ en particular de aquellos que hoy en día ni siquiera alcanzan los niveles básicos de supervivencia (Lanz, 2004).

La lectura y análisis de la bibliografía referida en párrafos anteriores sobre este tema, permitió inventariar los elementos que están presentes en todas las reflexiones acerca del mismo, entre ellos los saberes locales, la capacidad de innovación local, la organización en redes, la 
cultura democrática, la participación de todos los actores locales, la iniciativa productiva a lo interno, la visión clara de futuro, la independencia del centro, la inversión en el sistema productivo, la sustentabilidad económica, cultural en el tiempo. Es así como Leal (2013) y Gherab (2012), insisten que entre los factores determinantes de la innovación, está el factor educativo, y en sus aportes hacen referencia a la baja capacidad en el uso de las tecnologías de información y comunicación (tic's), así como del poco apoyo del Estado. Estos elementos están contemplados según el nivel de pertinencia en las distintas dimensiones del sistema diseñado.

\section{La emergencia del subsistema de evaluación heurística}

El subsistema de autoevaluación heurística, componente sugerido para el sistema de innovación tecnológica estructurado bajo los principios de desarrollo endógeno, pretende ser una orientación teórico-metodológica inspirada en la teoría de sistemas sociales de Luhmann (2007), cuya finalidad es permitir a los actores sociales involucrados en el desarrollo local, incorporarse en un proceso de auto aprendizaje continuo que garantice la sostenibilidad del sistema.

El valor agregado del subsistema lo constituye la propuesta de relaciones entre sus partes estructurales a través del aprendizaje continuo.

El subsistema gestiona la evaluación crítica permanente a lo interno del sistema con estrategias gerenciales que conducen a la configuración de consensos representados en símbolos signos y nuevos significados.
Supone la planificación de un programa de capacitación permanente, que garantice la participación de los actores sociales con mecanismos estructurados, claros y sistematizados. Permite reconocer la importancia de los recursos intangibles generados por el conocimiento, y su adecuada gestión versus la necesidad de grandes recursos materiales. En los aportes del Consejo Nacional de Innovación para la Competitividad (CNIC, 2010), encargado de elaborar la agenda de innovación y competitividad para el decenio 2010-2020, se informa sobre la situación del capital humano como el sector que representa el mayor desafío en el proceso de innovación. Esta afirmación es compartida también por Delgado y Navas (2008), en sus aportes sobre el capital intelectual como eje fundamental en los procesos de innovación, sobre todo en Iberoamérica, como consecuencia de las coincidencias culturales que comparten.

El subsistema se nutre teóricamente de la negación de la premisa de las explicaciones causales y lineales para intervenir la realidad, acusando la falta de resultados esperados. Así mismo reconoce la diversidad y la particularidad de los contextos y su relación con los entornos. Reconoce también, la complejidad de la realidad social y debería estar o ser construida y evaluada por quienes la comparten. Desde el punto de vista metodológico reconoce la ausencia de partes en absoluto, entendido con la afirmación de que: parte es un mero patrón dentro de una red inseparable de red de relaciones (Capra, 2005).

El subsistema de autoevaluación heurística componente del sistema de innovación tecnológica industrial sostenible, estructurado bajo los 
Sistema de innovación tecnológica desde la perspectiva del desarrollo...

Benavides de Finol, Luisa, Guzmán Parra, Vanesa y Vila Oblitas, José

principios de desarrollo endógeno, se configura conjuntamente con otros subsistemas incluido el medioambiental, pero posee muy poco conocimiento de la red de relaciones que son las que definen el funcionamiento del sistema.

Para que el sistema de innovación tecnológica sostenible, estructurado bajo los principios de desarrollo endógeno, genere insumos que soporten el proceso de toma de decisiones acertadas en el sistema, y apalanque el aprendizaje continuo a partir de las competencias que identifiquen los indicadores adecuados para la evaluación, debe conocer ampliamente la dinámica de la red de relaciones entre los subsistemas.

\section{Los ámbitos interno y externo}

El modelo teórico propuesto en este trabajo ha sido concebido a partir del principio de que la realidad social no se puede explicar en forma lineal. Se parte de la premisa de que el conocimiento no es continuo, tampoco es uniforme, no es ordenado, no corresponde a un esquema predeterminado y es muy complejo. (Capra, 2005).

Este enfoque lleva a comprender que para que funcione la innovación local se hace imprescindible visualizarlo de forma sistémica, es decir, el fenómeno con sus componentes y sus vinculaciones con los entornos tanto el externo, expresado en los patrones de interacción entre los grupos e individuos, y los internos dirigidos a las iniciativas innovación, los actores locales y las empresas.

Las vinculaciones requieren para el funcionamiento adecuado del sistema en general, componentes manejables directamente desde el ámbito interno y tener capacidad de propiciar acciones para la acción de otros sujetos fuera de este ámbito. Tales componentes dependen del apoyo de otros actores incluyendo el Estado. Según los estudiosos del tema los fracasos en la aplicación de las políticas en Venezuela vienen dados por la falta de mecanismos formales de articulación, entre el sector productivo local y las redes locales a la economía nacional, así también como, el desmantelamiento de la descentralización y debilitamiento de los gobiernos locales (Avalos, entrevista 2012).

Es así como la propuesta de un sistema de innovación nacional, como política pública de Estado tal como se ha venido mencionando, se convierte en el primer paso para poder emprender la aplicación de un modelo alternativo de desarrollo local.

Este sistema se diseña tomando en cuenta dos ámbitos: el externo y el interno y se desarrolla en tres dimensiones: la política, la económica y la tecnológica y cultural portadoras de los principios sustentadores del desarrollo endógeno (Vázquez- Barquero, 1997).

De allí que la innovación tecnológica en el marco del desarrollo endógeno estaría conformada por factores de índole externos, que se concretan en el tejido social constituido por un patrón de interacción entre individuos que promueven una estructura articuladora para la consolidación de un ambiente propicio para el fomento de iniciativas de innovación. Debe poseer además un marco institucional regulador promotor de una diferenciación territorial favorable para la materialización de estas iniciativas.

En lo relativo al ámbito interno las actividades propias de innovación deben también tener unas características orientadas hacia la invención y la adopción, fortalecidas éstas, por el desarrollo de las capacidades de 
resolución de problemas locales con un amplio conocimiento de la zona, el respeto a las vocaciones locales, en el proceso productivo, amén de los compromisos con su propio entorno, acompañado con el fortalecimiento de la identidad cultural.

El último factor es el actor, léase emprendedor o simplemente actor involucrado en el proceso de innovación, el cual debe poseer rasgos personales diferenciadores y una eficiencia productiva, capaz de mantener una alta expectativa en torno a las actividades que realiza, por lo que el sistema requiere un subsistema referido al actor, sus rasgos psicosociales y culturales para gestionar la autoestima e identidad nacional.
Arocena (2002), piensa que en el caso de los temas de los sistemas de innovación, habría que tomar en cuenta lo interdisciplinario, complejo y multifactorial que combina no solo una perspectiva histórica sino estructural con uno orientado a los actores, los involucrados.

En el diagrama 1, se describe el ámbito externo, las dimensiones y los indicadores que miden el proceso general de innovación local. En el diagrama 2, se describe el ámbito interno también con las dimensiones y los indicadores que según la investigación explican el proceso y forman parte de los componentes del sistema de innovación bajo los principios de desarrollo endógeno.

\section{Diagrama 1}

\section{Contexto externo del sistema nacional de innovación local}

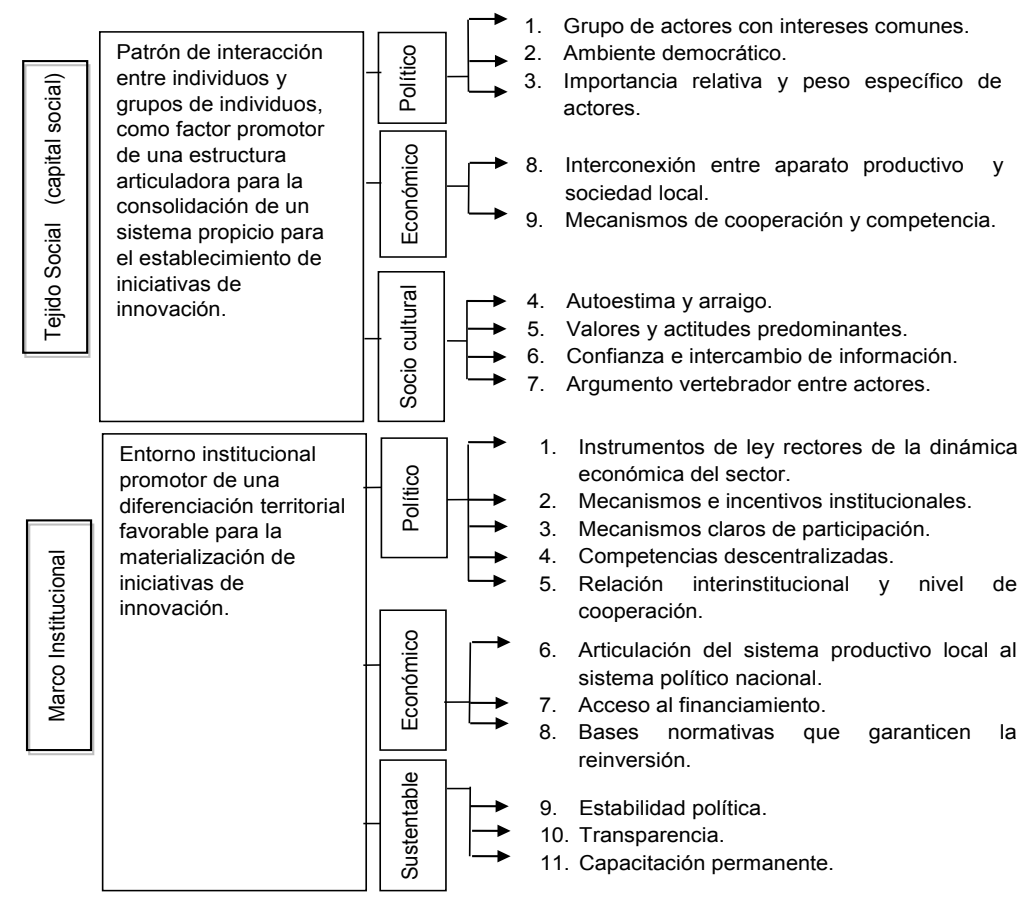

Fuente: Benavides (2012) 


\section{Diagrama 2}

\section{Contexto interno, del sistema nacional de innovación local}

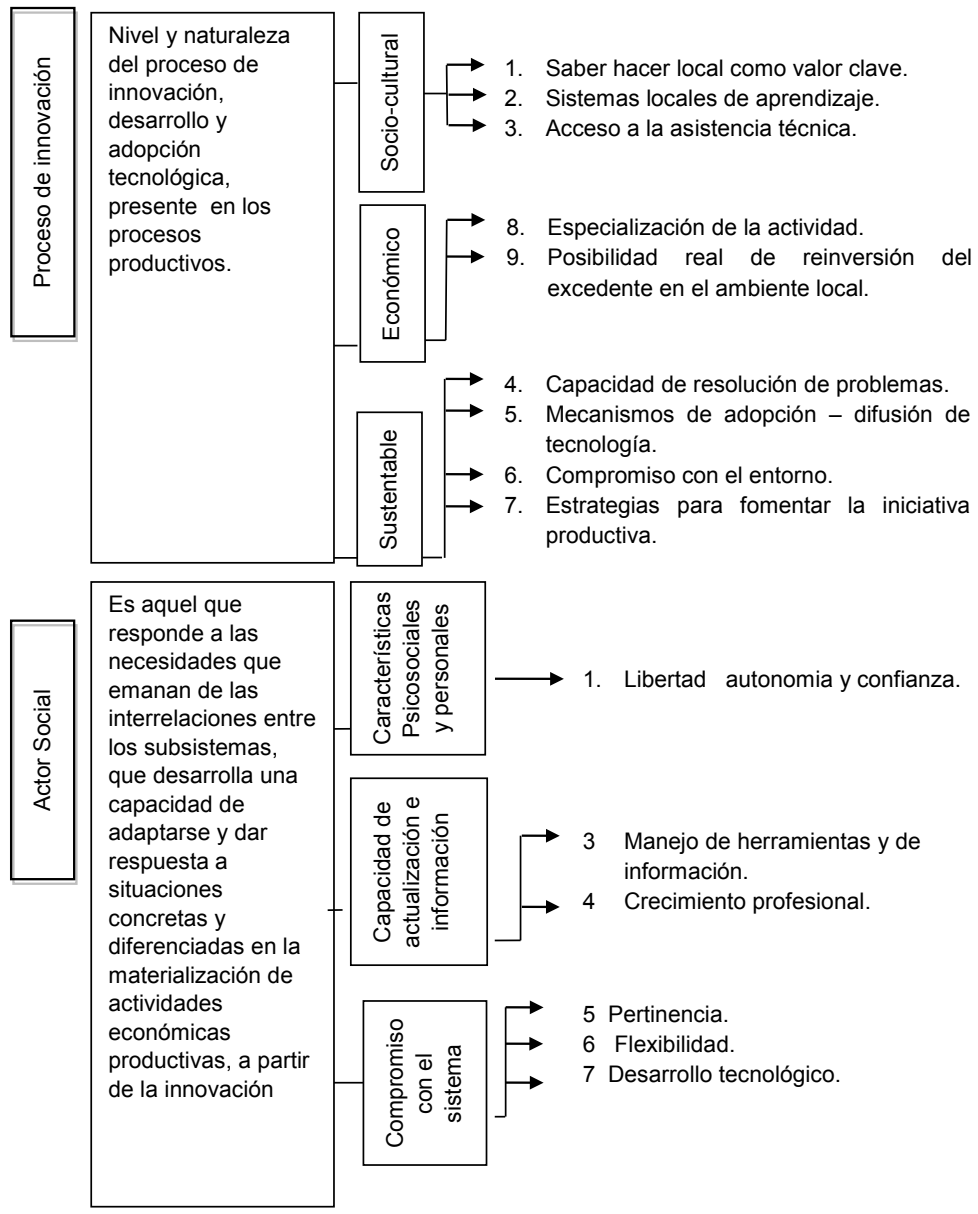

Fuente: Benavides (2012)

El actor social es uno de los componentes clave en el proceso de innovación bajo los principios de desarrollo endógeno. De allí la necesidad de incluir en los elementos constitutivos del sistema rasgos psicosociales del emprendedor (Benavides, 2012). Esto debe contribuir a mantener un proceso de aprendizaje permanente que permita los cambios y dinamice el sistema. En el diagrama 3 se vinculan los rasgos del emprendedor con los subsistemas. 


\section{Diagrama 3 \\ Rasgos del emprendedor y el sistema nacional de innovación. \\ Perfil \\ Sub-sistemas}

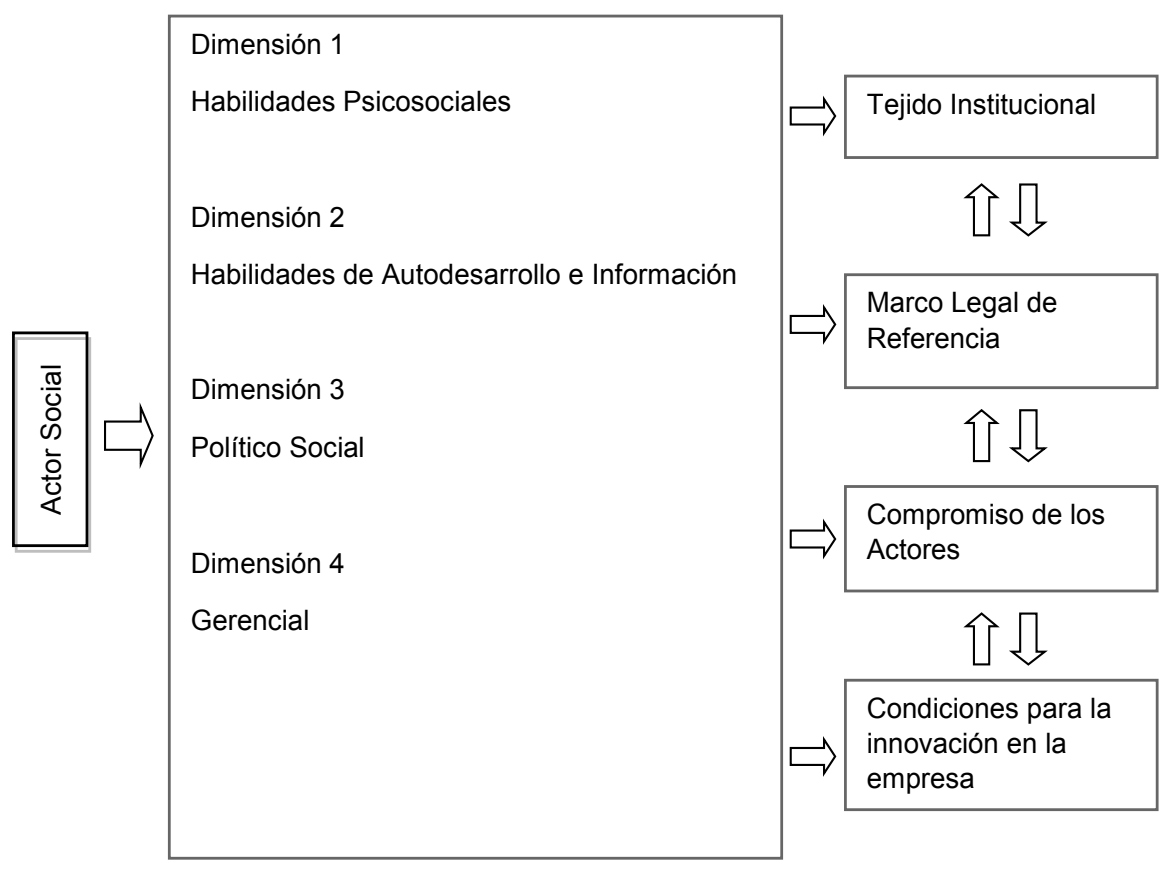

Fuente: Benavides (2012)

La innovación tecnológica resulta un tema de actualidad y sigue siendo objeto de estudio de diversas disciplinas. Desde la perspectiva del desarrollo ha sido tratada en este estudio, cuya esencia logró vincular la innovación tecnológica con el desarrollo desde un punto de vista holístico, que obliga a considerar el contexto en el cual se produce. Los resultados más relevantes de este trabajo de investigación se sintetizan en: La innovación tecnológica local tiene un carácter territorial, lo esto significa la estrecha vinculación a un entorno particular, esta condición le permite preservar la integridad cultural
(Buarque, 1999 citado por Boissier, 2009).

Los procesos de innovación tecnológica local están fundamentados en la integridad cultural asegurando, su desarrollo en un entramado social potenciador en la creación de redes de producción y consumo. La innovación tecnológica en su carácter territorial, integrador cultural y propiciador de redes de producción y consumo potencia la organización de la producción, la productividad y la acumulación de capital (Jaua, 2005).

La tradición productiva de cada localidad y el impulso de las relaciones 
Sistema de innovación tecnológica desde la perspectiva del desarrollo...

Benavides de Finol, Luisa, Guzmán Parra, Vanesa y Vila Oblitas, José

sociales de cooperación y competencia favorece las mejoras y desarrolla capacidades en los sistemas de empresas locales. Crea la capacidad de la autoconstrucción de los mismos sistemas a través de la autocrítica y la autoevaluación (Luhmann, 2007). Los procesos de innovación tecnológica local, son sustentables en la medida que garantizan su permanencia en el tiempo.

El estudio, da cuenta de dos hallazgos fundamentales. El primero, es que los procesos que se están dando en el país no son suficientes en magnitud y profundidad para hablar de desarrollo local $y$, en segundo lugar, que la instauración de un sistema que entienda el problema de la innovación tecnológica local como propiciador del proceso es de suma urgencia (Avalos, entrevista 2012).

\section{Conclusiones}

La reflexión teórica permitió identificar los elementos constitutivos de un sistema de innovación tecnológica, bajo los principios de desarrollo endógeno. Tal como se refiere en el texto, los conceptos de desarrollo endógeno y desarrollo local son trabajados indistintamente por los autores referenciados. Parece entonces que hablar de desarrollo endógeno y local es lo mismo sobre todo en lo que plantea Vázquez-Barquero (1997) que se desarrolla en pequeñas unidades territoriales.

La innovación tecnológica local, bajo los principios de desarrollo endógeno se produce en tres planos el sociocultural en el que se registran principios tales como: utilización de saberes locales para resolver problemas en la zona, accesibilidad a la formación cultural, actualización, arraigo, compromiso, transparencia, identidad cultural y autoestima.

En el plano político se requiere que los procesos se den de manera democrática y descentralizada, participativa con decisiones consensuadas y, en el plano económico, debe procurar la especialización de la actividad económica para facilitar la conformación del entramado de redes de producción y consumo, la capacidad de ganancia y de reinversión en la zona y un aparato legal capaz de garantizar su sustentabilidad, todo ello con la finalidad de mejorar la calidad de vida de todos los habitantes de la zona.

\section{Referencias bibliográficas}

\section{Albornoz, Mario (2002), Política científica y tecnológica. Una visión desde América Latina. Revista Iberoamericana de Ciencia, Tecnología, Sociedad e Innovación. $\quad \mathrm{N}^{\circ}$ 1, Septiembre- Diciembre 2002. Consultado el 28 de Febrero de 2006, en: http://www.oei. es/revistactsi/numero1/albornoz.htm}

Arocena, Rodrigo. (2002), Sistemas de innovación y países en desarrollo. Sudesca Research. Departament of Business Studies. $250 \mathrm{pp}$

Benavides, Luisa (2012), El perfil de la innovación en el Estado Lara. Universidad Centro Occidental Lisandro Alvarado CDCHT. Barquisimeto.

Boissier, Sergio (2005), ¿Y si el desarrollo fuese una emergencia sistémica? Revista Reforma y Democracia, CLAD, $n^{\circ}$ 27: Octubre 2005. Consultado el 15-04-2006 en: http://www.clad.org.ve.rev27boissier. pdf.

Boissier, Sergio (2009), Sinergia e innovación local. Revista Semestre Económico. Vol. 12, N 24 Edición especial pp 21-35 Oct 2009 MedellínColombia 
Calvelo, Manuel (2001), El Desarrollo Endógeno, Autogestionado y Sustentable. España Edt Runa

Cabello, Alejandra y Ortiz, Edgar (2013), Políticas públicas de innovación tecnológica y desarrollo: teoría y propuesta de educación superior. Convergencia. Revista de Ciencias Sociales. Vol. $20, n^{\circ}$. 61, enero-abril de 2013, pp. 135-172.

Capra, Fritjof (2005), La trama de la vida. Madrid. Editorial Anagrama.

Corvalán, Stella y Ferreira, María (2003), Desarrollo Local. Una metodología de la participación. Libros Madrid, Arce.

Consejo Nacional de Innovación para la Competitividad (CNIC) (2010), Avances, desafíos y propuestas en Capital Humano. Consultado el 01-02-2014 en:http://bibliotecacnic. ch/content/view/916114/avancesdesafios-y-propuestas-en-capitalhumano/html\#content-top.

Cuervo, Luis, Ramírez, Carlos y Silva, Iván (2009), Economía y territorio en América Latina y el Caribe. Desigualdades Políticas. Publicaciones de Naciones Unidas ( C.E.P.A.L) Impreso en Chile

Delgado, Miriam y Navas López, José (2008), La innovación tecnológica desde el marco del capital intelectual. Cuaderno de Trabajo $n^{\circ} 04$. Escuela Universitaria de Estadística. Universidad Complutense de Madrid. 38 pp. Consultado el 20-02-2014 en: http://estudiosestadisticos.ucm. es/data/cont/docs/12-2013-02-06CT04_2008.pdf.

Gherab, Karim (2012), La innovación tecnológica. ¿Cómo cambian las conductas? Nueva Revista. $n^{\circ} 140$, septiembre de 2012. Consultado el 20-02-2014 en: http:// www.nuevarevista.net/articulos/ la-innovacion-tecnologica-comocambian-las-conductas.

Jaua, Elías (2005), Nuevo Modelo Socio-Productivo y Desarrollo
Endógeno. Caracas. Ediciones del MINEP-INCE.

Lanz, Carlos. (2004), La propuesta de Desarrollo Endógeno tiene que partir de otros valores. Rebelión, 1 de Marzo de 2004. Consultado el el 15-04- 2004 en http://rebelion.org/ noticia. php?id=11965.

Leal, Miraidy (2013), Factores determinantes de la innovación tecnológica de las PYMES del sector confección. Revista de Ciencias Sociales (RCS) Vol. XVIII, $\mathrm{n}^{\circ} 3$, julio - septiembre de 2012, pp. 540-552.

López Doval, Jaime (1997), Artículo sobre Vázquez-Barquero. Revista Bibliográfica de Geografía y Ciencias Sociales. Universidad de Barcelona. cissn1138-9796. 10 de Marzo 1997. Na13

Luhmann, Niklas (2007), La sociedad de la sociedad. México. Editorial Herder

Martínez Lillo, Pedro (2012), Cooperación internacional y desarrollo local Editorial La Catarata. Colombia.

Ministerio de Planificación y Desarrollo Social (2005), Plan de Desarrollo. Caracas. pp.75.

Ochoa, Alejandro. (2005), Desarrollo Endógeno un debate impostergable. Consultado el 2803-2013 en: http://www.aporrea.org/ actualidad/a15217.html

Organización de Estados Iberoamericanos para la Educación, la Ciencia y la Cultura (2012), Ciencia, tecnología e innovación para el desarrollo y la cohesión social. Programa iberoamericano en la década de los bicentenarios. Coordinador Mario Albornoz. 95 pp.

Presidencia de la República (2005), Decreto $N^{\circ}$ 3895. Desarrollo endógeno Empresas de producción social...

Quintero, Johana y López, Leonel (2012), Estrategia de innovación tecnológica en las organizaciones. Revecitec URBE, Vol. $3, \mathrm{n}^{\circ}$. 1, julio - diciembre de 2012. Consultado el 20-02-14, 
Sistema de innovación tecnológica desde la perspectiva del desarrollo... Benavides de Finol, Luisa, Guzmán Parra, Vanesa y Vila Oblitas, José

en: http://www.publicaciones.urbe. edu/index.php/revecitec/article/ viewArticle/1514/3421.

Schumpeter, Joseph (1934), The theory of economic development. Cambridge. Harvard University Press.

Stiglitz, Joseph. E (2013), El informe Stizlitz. Editorial RBA Libros. Madrid

Slee, Bill (2003), Endogenous Development; A Concept in Search of a Theory. CIHEAM-Options Mediterrannennes, Ser $A / n^{\circ} 23$. Consultado el 1 de febrero de 2011 de http://ressources.cieham. org/om/pdf/a23/Cl000373.pdf

Touraine, Alain (2001), América Latina. Política y sociedad. Madrid. Editorial Espasa-Calpe.
Vega, Marco (2012), Aspectos y avances en ciencia, tecnología e innovación. Revista Polis, $n^{\circ} 33$. Consultado el 01-02-2014 en: http://polis.revues. org/8619

Vázquez- Barquero, José (1997), La política económica global La respuesta de las ciudades a los desafíos del ajuste productivo. Madrid- Editorial Pirámide 1993 332pg

\section{Entrevistas}

Avalos, Ignacio. Sociólogo experto en el tema innovación. Ex presidente de CONICIT, Caracas, Venezuela. Entrevista 13 de Julio de 2012. 